\title{
Convergence of Bogoliubov's Method of Renormalization in Momentum Space
}

\author{
W. ZIMMERMANN ${ }^{\star}$ \\ Institut des Hautes Etudes Scientifiques, Bures sur Yvette \\ and \\ Istituto di Fisica Teorica, Mostra d'Oltremare, Napoli
}

Received June 25, 1969

\begin{abstract}
Bogoliubov's method of renormalization is formulated in momentum space. The convergence of the renormalized Feynman integrand is proved by an application of the power counting theorem.
\end{abstract}

\section{Introduction}

A general theory of renormalization has been developed by Bogoliubov for arbitrary local and invariant interactions. It was shown by Hepp that the renormalized Feynman integrals constructed according to Bogoliubov's rules converge to well defined distributions when the regularization is removed [1-4].

In a recent paper [5] a different formulation of Bogoliubov's method was used which works in momentum space and does not refer to a regularization. The starting point of this approach is the integrand $I_{\Gamma}$ of the unrenormalized Feynman integral

$$
J_{\Gamma}\left(p_{1} \cdots p_{r}\right)=\lim _{\varepsilon \rightarrow+0} \int d k_{1} \cdots d k_{m} I_{\Gamma}\left(k_{1} \cdots k_{m}, p_{1} \cdots p_{r}\right)
$$

in momentum space. The integrand $R_{\Gamma}$ of the finite part of (1.1)

$$
F_{\Gamma}\left(p_{1} \cdots p_{r}\right)=\lim _{\varepsilon \rightarrow+0} \int d k_{1} \cdots d k_{m} R_{I}\left(k_{1} \cdots k_{m}, p_{1} \cdots p_{r}\right)
$$

is defined as a rational function of the internal and external momenta by substracting appropriate counter terms from $I_{\Gamma}$. The method is thus an extension of the original work of Dyson and Salam [6-8] ${ }^{1}$. For handling the overlapping divergencies Bogoliubov's combinatorial technique is used which applies to renormalizable as well as non-renormalizable theories.

* On leave of absence from Courant Institute of Mathematical Sciences, New York University, New York.

${ }^{1}$ For some references of other methods of renormalization see [9-12]. 
In this paper the convergence of the renormalized integral (1.2) is proved by a simple application of the power counting theorem [13-15]. The main problem will be to verify the hypothesis of the power counting theorem, i.e. to check that the dimension of (1.2) and any sub-integral along an arbitrary hyperplane in $k$-space has negative dimension. The power counting theorem for Minkowski metric then implies that the integrals are absolutely convergent for $\varepsilon>0$ and yield co-variant distributions in the limit $\varepsilon \rightarrow 0$ [15].

In Section 2 the definition of the finite part (1.2) is discussed. An explicit formula for $R_{\Gamma}$ is derived in Section 3. Section 4 contains the proof that the renormalized Feynman integral meets the requirements of the power counting theorem.

\section{The Finite Part of an Arbitrary Feyman Integral}

We consider a Feynman diagram $\Gamma$ with $N$ vertices $V_{1}, \ldots, V_{N}$. The lines connecting the vertices $V_{a}, V_{b}$ will be denoted by $L\left(V_{a}, V_{b}, \sigma\right)$ or $L_{a b \sigma}(\sigma=1, \ldots, v(a b)) . V_{a}, V_{b}$ are called endpoints of $L_{a b \sigma}$. Lines connecting a vertex with itself are excluded, $(a=b)$. Each vertex is supposed to be endpoint of at least one line. No restriction is placed on the number of lines joining at a vertex.

$\mathscr{L}(\Gamma)$ denotes the set of lines, $\mathscr{V}(\Gamma)$ the set of vertices.

$$
\sum_{a b \sigma} \Gamma, \prod_{a b \sigma} \Gamma
$$

denote sum or product resp. over all lines $L_{a b \sigma}$ of the diagram $\Gamma$.

$$
\sum_{a}, \prod_{a}
$$

denote sum or product resp. over all vertices $V_{a}$ of the diagram $\Gamma$.

To each line $L_{a b \sigma}$ we assign an internal momentum

$$
l_{a b \sigma}=-l_{b a \sigma} .
$$

To each vertex $V_{a}$ a momentum $q_{a}$ is assigned. In general the $q_{a}$ will be linear combinations of external momenta $p_{1}, \ldots, p_{r}$

$$
q_{a}=q_{a}\left(p_{1} \cdots p_{r}\right) .
$$

The internal and external momenta are subject to the relation of momentum conservation at each vertex

$$
\sum_{b \sigma}^{a} r l_{a b \sigma}=q_{a} \quad a=1, \ldots, N .
$$

Here $\sum_{b \sigma}^{a} \Gamma$ denotes the sum over all lines $L_{a b \sigma}$ of the diagram $\Gamma$ having $V_{a}$ as one of its endpoints. As consequence of (2.1), (2.3) the external mo- 
menta must satisfy momentum conservation

$$
\sum_{a} r_{v} q_{a}=0 \quad v=1, \ldots, c
$$

with $\Gamma_{1}, \ldots, \Gamma_{\mathrm{c}}$ denoting the connected components of $\Gamma$.

To each line $L_{a b \sigma}$ we assign a propagator

$\Delta_{F}^{a b \sigma}=P_{a b \sigma}\left(l_{a b \sigma}\right)\left(l_{a b \sigma}^{2}-\mu_{a b \sigma}^{2}+i \varepsilon\left(\boldsymbol{l}_{a b \sigma}^{2}+\mu_{a b \sigma}^{2}\right)\right)^{-1}, \varepsilon>0, \mu_{a b \sigma}^{2}>0$

where $P_{a b \sigma}$ denotes a polynomial in the components of $l_{a b \sigma}$. To each vertex $V_{a}$ we assign a polynomial

$$
P_{a}=P_{a}\left(l_{a b_{1} 1}, \ldots, l_{a b_{\tau} v\left(a b_{\tau}\right)}\right)
$$

in the components of the vectors $l_{a b 1}, \ldots$ Here $V_{b_{1}}, \ldots, V_{b_{\tau}}$ denote the vertices which are connected to $V_{a}$ by an internal line. With these insertion rules the corresponding unrenormalized integral becomes

$$
J\left(p_{1}, \ldots, p_{r}\right)=\lim _{\varepsilon \rightarrow+0} \int d k_{1} \cdots d k_{m} \prod_{a b \sigma} \Delta_{F}^{a b \sigma} \prod_{a} P_{a} .
$$

The internal momenta in $\Delta_{F}^{a b \sigma}, P_{a}$ (Eq. (2.5-2.6)) are of the form

$$
l_{a b \sigma}=k_{a b \sigma}+q_{a b \sigma} .
$$

The $q_{a b \sigma}$ are linear combinations

$$
q_{a b \sigma}=q_{a b \sigma}\left(q_{1}, \ldots, q_{N}\right)
$$

of $q_{1}, \ldots, q_{N}$ and form a particular solution of

$$
\sum_{b \sigma}^{a} q_{a b \sigma}=q_{a}, \quad q_{a b \sigma}+q_{b a \sigma}=0 .
$$

The $q_{a b \sigma}$ are called basic internal momenta. The $k_{a b \sigma}$ are linear combinations

$$
k_{a b \sigma}=k_{a b \sigma}\left(k_{1}, \ldots, k_{m}\right)
$$

of the integration variables $k_{1}, \ldots, k_{m}$ and represent the general solution of the homogeneous equations

$$
\sum_{b \sigma}^{a} k_{a b \sigma}=0, \quad k_{a b \sigma}+k_{b a \sigma}=0 .
$$

$m$ of the forms $k_{a b \sigma}$ are chosen as independent four vectors $k_{1}, \ldots, k_{m}$. We introduce the following abbreviations

$$
k=\left(k_{1}, \ldots, k_{m}\right), q=\left(q_{1}, \ldots, q_{N}\right), p=\left(p_{1}, \ldots, p_{r}\right) .
$$

$K$ denotes the set

$$
K=\left\{k_{a b \sigma}\right\}_{L_{a b \sigma} \in \mathscr{L}(\Gamma)},
$$


of four vectors $k_{a b \sigma}$ satisfying (2.12). Equations (2.2), (2.11) are written as

$$
q=q(p), K=K(k) \text {. }
$$

In this notation the Feynman integral becomes

$$
J(p)=\lim _{\varepsilon \rightarrow+0} \int d k_{1} \cdots d k_{m} I_{\Gamma}(K(k), q(p))
$$

where

$$
I_{\Gamma}(K, q)=\prod_{a b \sigma} \Delta_{F}^{a b \sigma} \prod_{a} P_{a} .
$$

$\Delta_{F}^{a b \sigma}, P_{a}$ are given by (2.5-2.6) with the substitutions

$$
l_{a b \sigma}=l_{a b \sigma}(K q)=k_{a b \sigma}+q_{a b \sigma}(q) .
$$

In this section the finite part of (2.16) will be defined in the form ${ }^{2}$

$$
F_{\Gamma}(p)=\lim _{\varepsilon \rightarrow+0} \int d k_{1} \cdots d k_{m} R_{\Gamma}(K(k), q(p))
$$

where the modified integrand $R_{\Gamma}(k q)$ is obtained from the original integrand $I_{\Gamma}(k q)$ by a suitable number of subtractions. Unfortunately the definition of $R_{r}$ will depend on the choice of the basic internal momenta $q_{a b \sigma}(q)$. Though the final integral (2.19) is the same for a large class of basic momenta, we will - for the sake of definiteness - make a unique choice of $q_{a b \sigma}$ in the definition of the finite part. To this end we define the canonical momenta as that solution of (2.10) for which the quadratic form

$$
\sum_{a b \sigma} q_{a b \sigma}^{2}
$$

is stationary under the constraints $(2.10)$. With the Lagrange multipliers $u_{1}, \ldots, u_{N}$ of the constraints (2.10) the $q_{a b \sigma}$ become uniquely determined by

$$
\begin{gathered}
q_{a b \sigma}=u_{a}-u_{b}, \\
\sum_{b \sigma}^{a} q_{a b \sigma}=q_{a}, \quad \sum_{a} \Gamma_{v} q_{a}=0 .
\end{gathered}
$$

These are $N-c$ independent equations for the $N-c$ independent differences $u_{a}-u_{b}$ if $c$ is the number of connected components of $\Gamma$.

We introduce some combinatorial concepts which will be needed later on.

Let $\mathscr{L}(\Delta)$ denotes the set of lines and $V(\Delta)$ denote the set of vertices of a diagram $\Delta$. To any set $\mathscr{L} \cong \mathscr{L}(\Gamma)$ we define a subdiagram $\Delta$ of $\Gamma$ by the lines $L \in \mathscr{L}$ and the vertices which are endpoints of a line in $\mathscr{L}$.

2 The integral does not depend on the choice of $k_{1}, \ldots, k_{m}$. For, any two sets $k_{1}, \ldots, k_{m}$ and $k_{1}^{\prime}, \ldots, k_{m}^{\prime}$ of $m$ linearly independent internal momenta $k_{a b \sigma}$ are related by an orthogonal transformation. 
We say that the diagram $\Delta$ is spanned by the set $\mathscr{L}$ of lines. The following definitions concern subdiagrams of a given diagram $\Gamma$. We define $\Delta=\Delta_{1} \cap \Delta_{2}$ as the diagram spanned by

$$
\mathscr{L}(\Delta)=\mathscr{L}\left(\Delta_{1}\right) \cap \mathscr{L}\left(\Delta_{2}\right) .
$$

$\Delta_{1}$ is called a subdiagram of $\Delta_{2}$, i.e. $\Delta_{1} \subseteq \Delta_{2}$ if $\mathscr{L}\left(\Delta_{1}\right) \subseteq \mathscr{L}\left(\Delta_{2}\right)$. If $\Delta_{1} \subseteq \Delta_{2}$, the diagram $\Delta_{2} \backslash \Delta_{1}$ is defined as the diagram spanned by $\mathscr{L}\left(\Delta_{2}\right) \backslash \mathscr{L}\left(\Delta_{1}\right)^{3}$

Let $\delta$ be a subdiagram of $\Delta$ with connected components $\delta_{1}, \ldots, \delta_{c}$. We form the reduced diagram

$$
\bar{\Delta}=\Delta / \delta=\Delta / \delta_{1} \cdots \delta_{c}
$$

by contracting each line of $\delta$ to a point. More precisely the reduced diagram $\Delta$ is defined by

$$
\mathscr{L}(\bar{\Delta})=\mathscr{L}(\Delta) \backslash \mathscr{L}(\delta), \mathscr{V}(\bar{\Delta})=\mathscr{V}(\Delta) \backslash \mathscr{V}(\delta) \cup\left\{\bar{V}_{1}, \ldots, \bar{V}_{c}\right\} .
$$

Here

$$
\bar{V}_{1}=\mathscr{V}\left(\delta_{1}\right), \ldots, \bar{V}_{c}=\mathscr{V}\left(\delta_{c}\right)
$$

serve as new vertices of $\bar{\Delta}$ replacing the vertices of the reduced diagrams $\delta_{1}, \ldots, \delta_{c}$. Two vertices $V, V^{\prime} \in \mathscr{V}(\Delta) \backslash \mathscr{V}(\delta)$ are connected in $\bar{\Delta}$ by the same lines as in $\Delta . V \in \mathscr{V}(\Delta) \backslash \mathscr{V}(\delta)$ and $\bar{V}_{a}$ are in $\bar{\Delta}$ connected by all lines of $\mathscr{L}(\Delta) \backslash \mathscr{L}(\delta)$ which in $\Delta$ connect $V$ with any vertex of $\bar{V}_{a} \cdot \bar{V}_{a}$ and $\bar{V}_{b}$ are connected by all lines of $\mathscr{L}(\Delta) \backslash \mathscr{L}(\delta)$ which in $\Delta$ connect a vertex of $\delta_{a}$ with a vertex of $\delta_{b}$.

In the work that follows we will consider subdiagrams as well as reduced diagrams of our original Feynman diagram $\Gamma$. Let $\gamma \cong \Gamma$ be a subdiagram of $\Gamma . m(\gamma)$ denotes the number of independent internal momenta, $N(\gamma)$ the number of vertices of $\gamma$. The unrenormalized integrand $I_{\gamma}$ is defined by the same insertion rules as for $I_{\Gamma}$. Explicitly

$$
\begin{gathered}
I_{\gamma}\left(K^{\gamma} q^{\gamma}\right)=\prod_{a b \sigma} \Delta_{F}^{a b \sigma} \prod_{a} P_{a}, \\
K^{\gamma}=\left\{k_{a b \sigma}^{\gamma}\right\}_{L_{a b \sigma} \in \mathscr{L}(\gamma)}, \quad q^{\gamma}=\left\{q_{a}^{\gamma}\right\}_{V_{a} \in \mathscr{V}(\gamma)} .
\end{gathered}
$$

$\Delta_{F}^{a b \sigma}, P_{a}$ are given by (2.5-2.6) with the substitutions

$$
l_{a b \sigma}=l_{a b \sigma}^{\gamma}\left(K^{\gamma}, q^{\gamma}\right)=k_{a b \sigma}^{\gamma}+q_{a b \sigma}^{\gamma}\left(q^{\gamma}\right) .
$$

Here $q_{a b \sigma}^{\gamma}$ denote the canonical momenta defined in reference to the subdiagram $\gamma$. I.e. $q_{a b \sigma}^{\gamma}$ is that solution of

$$
\sum_{a b \sigma}^{a} q_{a b \sigma}^{\gamma}=q_{a}, q_{a b \sigma}^{\gamma}+q_{b a \sigma}^{\gamma}=0, V_{a} \in \mathscr{V}(\gamma)
$$

\footnotetext{
${ }^{3} A \backslash B$ denotes the difference of the two sets $A, B$.
} 
for which

$$
\sum_{a b \sigma}\left(q_{a b \sigma}^{\gamma}\right)^{2}
$$

is stationary. The four vectors $k_{a b \sigma}^{\gamma}$ satisfy

$$
\sum_{a b \sigma}^{a} k_{a b \sigma}^{\gamma}=0, k_{a b \sigma}^{\gamma}+k_{b a \sigma}^{\gamma}=0, V_{a} \in \mathscr{V}(\gamma) \text {. }
$$

We next define $k_{a b \sigma}^{\gamma}, q_{a}^{\gamma}$ as linear combinations of $k_{a b \sigma}, q_{a}$ by requiring

$$
l_{a b \sigma}^{\gamma}\left(K^{\gamma} q^{\gamma}\right) \equiv l_{a b \sigma}(K q)
$$

for all $L_{a b \sigma} \in \mathscr{L}(\gamma)$. According to

$$
q_{a}^{\gamma}(K q)=\sum_{a b \sigma}^{a} l_{a b \sigma}(K q), k_{a b \sigma}^{\gamma}(K q)=l_{a b \sigma}(K q)-q_{a b \sigma}^{\gamma}(K q)
$$

the $k_{a b \sigma}^{\gamma}, q_{a}^{\gamma}$ are uniquely determined by this requirement. Equation (2.31) implies

$$
q_{a}^{\gamma}(K q)=-\sum_{a b \sigma}^{a} l_{\backslash \gamma} l_{a b \sigma}(K q)=-\sum_{a b \sigma}^{a} \Gamma \backslash \gamma\left(k_{a b \sigma}+q_{a b \sigma}(q)\right) .
$$

It can further be shown that the $k_{a b \sigma}^{\gamma}$ depend only on the $k_{a b \sigma}$. For the Eqs. (2.21) and (2.31) imply

$$
\begin{aligned}
q_{a b \sigma}(q)= & u_{a}-u_{b} \text { for } V_{a}, V_{b} \in \mathscr{V}(\gamma), \\
& \sum_{a b \sigma}^{a} q_{a b \sigma}(q)=q_{a}^{\gamma}(0, q) .
\end{aligned}
$$

These equations, however, determine the functions $q_{a b \sigma}^{\gamma}(0, q)$.

Accordingly

and

$$
q_{a b \sigma}^{\gamma}(0 q)=q_{a b \sigma}(q)
$$

$$
k_{a b \sigma}^{\gamma}(0 q)=l_{a b \sigma}(0 q)-q_{a b \sigma}^{\gamma}(0 q)=0
$$

which proves the assertion. Hence we have the result that $K^{\gamma}, q^{\gamma}$ are linear combinations of the form

$$
K^{\gamma}=K^{\gamma}(K), q^{\gamma}=q^{\gamma}(K q) .
$$

With the substitutions (2.26), (2.34) the unrenormalized integral $I_{\gamma}$ becomes a function of $K$ and $q$.

Let $\mu$ be a subdiagram of $\gamma \subset \Gamma$. In an analogous way we introduce the function

$$
K^{\mu}=K_{\gamma}^{\mu}\left(K^{\gamma}\right), q^{\mu}=q_{\gamma}^{\mu}\left(K^{\gamma} q^{\gamma}\right)
$$

by the requirement

$$
l_{a b \sigma}^{\mu}\left(K^{\mu} q^{\mu}\right) \equiv l_{\alpha b \sigma}^{\gamma}\left(K^{\gamma}, q^{\gamma}\right) .
$$

For later use we note the relation corresponding to (2.32)

$$
q_{a}^{\mu}\left(K^{\gamma} q^{\gamma}\right)=-\sum_{a b \sigma}^{a}{ }_{\gamma \backslash \mu}\left(k_{a b \sigma}^{\gamma}+q_{a b \sigma}^{\gamma}\left(q^{\gamma}\right)\right)
$$


If $\gamma$ is proper we define the dimension $d(\gamma)$ by

$$
d(\gamma)=\sum_{a b \sigma} d\left(L_{a b \sigma}\right)+\sum_{a} d\left(V_{a}\right)+4 m(\gamma) .
$$

Here $d\left(L_{a b \sigma}\right)$ is the degree of the propagator corresponding to $L_{a b \sigma}$ with respect to the components of $l_{a b \sigma}, d\left(V_{a}\right)$ is the degree of the polynomial assigned to $V_{a}$. Apparently $d(\gamma)$ is the dimension of the unrenormalized Feynman integral of $\gamma$. Proper subdiagrams of dimension $d(\gamma) \geqq 0$ are called renormalization parts.

We next study reduced diagrams of $\Gamma$. Let $\gamma_{1}, \ldots \gamma_{c}$ be subdiagrams of $\Gamma$, proper and mutually disjoint. We consider the reduced diagram

$$
\bar{\Gamma}=\Gamma / \gamma_{1} \ldots \gamma_{c} .
$$

We define the unrenormalized integrand $I_{\bar{T}}$ by the same insertion rules as for $I_{\Gamma}$ except that the factor 1 is assigned to the reduced vertices $V_{1}, \ldots, V_{c}$. Explicitly

$$
I_{\bar{\Gamma}}(q K)=\prod_{a b \sigma} \Delta_{\bar{\Gamma}}^{a b \sigma} \prod_{a} P_{a}
$$

with $(2.5-2.6)$ and (2.18). Here $\prod_{a b \sigma}, \prod_{a}$ denotes the product over all lines $L_{a b \sigma}$ or vertices $V_{a}$ resp. of $\Gamma$ which do not belong to $\gamma_{1}, \ldots, \gamma_{c}$. Apparently (2.40) agrees with the definition (2.17) if applied to the reduced diagram $\bar{\Gamma}$.

After these preparations we now give the definition of the finite part of a Feynman integral. The integrand $R_{\Gamma}(K, q)$ of the finite part (2.19) is defined by

with

$$
R_{\Gamma}(K, q)=I_{\Gamma}(K, q)+\sum_{\gamma_{1} \ldots \gamma_{c}} I_{\Gamma / \gamma_{1} \ldots \gamma_{c}}(K, q) \prod_{\tau=1}^{c} O_{\gamma_{\tau}}\left(K^{\gamma_{\tau}}, q^{\gamma_{\tau}}\right)
$$

$$
K^{\gamma_{\tau}}=K^{\gamma_{\tau}}(K), q^{\gamma_{\tau}}=q^{\gamma_{\tau}}(K, q) .
$$

The sum extends over all sets $s=\left(\gamma_{1}, \ldots, \gamma_{c}\right)$ of renormalization parts of $\Gamma$ which are mutually disjoint

$$
\gamma_{\tau} \cap \gamma_{\sigma}=\theta \text { for } \tau \neq \sigma .
$$

This includes the case that $s$ consists of $\Gamma$ itself provided $\Gamma$ is a renormalization part. The functions $O_{\gamma}$ are recursively defined for every renormalization part $\gamma$ of $\Gamma$ by $O_{\gamma}\left(K^{\gamma} q^{\gamma}\right)$

with

$$
=-t_{q^{\gamma}}^{d(\gamma)}\left\{I_{\gamma}\left(K^{\gamma} q^{\gamma}\right)+\sum_{\gamma_{1} \ldots \gamma_{c}}^{\prime} I_{\Gamma / \gamma_{1} \ldots \gamma_{c}}\left(K^{\gamma} q^{\gamma}\right) \prod_{\tau=1}^{c} O_{\gamma_{\tau}}\left(K^{\gamma_{\tau}}, q^{\gamma_{\tau}}\right)\right\}
$$

$$
K^{\gamma_{\tau}}=K_{\gamma}^{\gamma_{\tau}}\left(K^{\gamma}\right), q^{\gamma_{\tau}}=q_{\gamma}^{\gamma_{\tau}}\left(K^{\gamma}, q^{\gamma^{\prime}}\right)
$$


$\sum^{\prime \gamma}$ denotes the sum over all sets $s=\left(\gamma_{1}, \ldots, \gamma_{c}\right)$ of renormalization parts $\gamma_{a} \neq \gamma$ of $\gamma$ which are mutually disjoint. The ' indicates that $s=\{\gamma\}$ is excluded in the sum. $t_{q}^{\nu_{\gamma}}$ applied to a function $F\left(q^{\gamma}\right)$ of $q_{1}^{\gamma}, \ldots, q_{M}^{\gamma}$ denotes the Taylor series in the components of the vectors $q_{i}^{?}$ up to the order $v$. This completes the definition of the finite part.

We further introduce a function $\bar{R}_{\gamma}$ by

$$
\bar{R}_{\gamma}\left(K^{\gamma} q^{\gamma}\right)=I_{\gamma}\left(K^{\gamma} q^{\gamma}\right)+\sum_{\gamma_{1} \ldots \gamma_{c}}^{\prime \gamma} I_{\Gamma / \gamma_{1} \ldots \gamma_{c}}(K q) \prod_{\tau=1}^{c} O_{\gamma_{\tau}}\left(K^{\gamma_{\tau}}, q^{\gamma_{\tau}}\right) .
$$

Apparently one has

$$
O_{\gamma}=-t_{q^{\gamma}}^{d(\gamma)} \bar{R}_{\gamma}
$$

$R_{\Gamma}$ is related to $\bar{R}_{\Gamma}$ by

$$
R_{\Gamma}=\bar{R}_{\Gamma}
$$

if $\Gamma$ is no renormalization part and

$$
R_{\Gamma}=\bar{R}_{\Gamma}+O_{\Gamma}=\left(1-t_{q}^{d}\right) \bar{R}_{\Gamma}
$$

if $\Gamma$ is a renormalization part.

The definition of the finite part can be generalized in various respect. First of all we remark that it is sometimes necessary to consider other sets of basic internal momenta besides of the canonical momenta. We give the appropriate definitions for a sufficiently general class of basic internal momenta.

Let $q_{a b \sigma}^{\gamma}$ be basic internal momenta given for every subdiagram $\gamma$ of $\Gamma$. We consider the set $S$ of all $q_{a b \sigma}^{\gamma}$. Again we can define $q_{a}^{\gamma}, k_{a b \sigma}^{\gamma}$ as linear combinations of $q, k$ requiring that

with

$$
l_{a b \sigma}^{\gamma}\left(K^{\gamma}, q^{\gamma}\right) \equiv l_{a b \sigma}(K, q)
$$

$$
\begin{aligned}
& l_{a b \sigma}^{\gamma}=k_{a b \sigma}^{\gamma}+q_{a b \sigma}^{\gamma}\left(q^{\gamma}\right), \\
& l_{a b \sigma}=k_{a b \sigma}+q_{a b \sigma}(q) .
\end{aligned}
$$

The set $S$ is called admissable if the momenta $K^{y}$ depend only on $K$ or $K^{\mu}$ resp.

$$
K^{\gamma}=K^{\gamma}(K), K^{\gamma}=K_{\mu}^{\gamma}\left(K^{\mu}\right) \text { for any } \mu \supset \gamma .
$$

The canonical internal momenta are an example of an admissable set of basic internal momenta ${ }^{4}$.

Let $\left\{q_{a b \sigma}^{\gamma}\right\}$ be a set of basic internal momenta for $\Gamma$. Then a set of basic internal momenta $\left\{\tilde{q}_{a b \sigma}^{\lambda}\right\}$ for the reduced diagram $\Gamma / \delta_{1} \ldots \delta_{c}$

${ }^{4}$ It can be shown that the finite part (2.19) is the same for any admissable set of basic internal momenta. 
may be introduced in the following way. For

Set

$$
L_{a b \sigma} \in \mathscr{L}(\lambda), \lambda \cong \Gamma / \delta_{1} \ldots \delta_{c}, \lambda=\gamma / \delta_{1} \ldots \delta_{c}, \gamma \cong \Gamma .
$$

$$
\tilde{q}_{a b \sigma}^{\lambda}=q_{a b \sigma}^{\gamma} .
$$

$\left\{\tilde{q}_{a b \sigma}^{\lambda}\right\}$ is called the set of basic internal momenta induced by $\left\{q_{a b \sigma}^{\gamma}\right\}$ in $\Gamma / \delta_{1} \ldots \delta_{c^{\prime}}$. If $\left\{q_{a b \sigma}^{\gamma}\right\}$ is admissable $\left\{\tilde{q}_{a b \sigma}^{\lambda}\right\}$ is also admissable. Apparently the function $I_{\bar{\Gamma}}(K, q)$ as defined by $(2.40)$ is constructed by using the basic internal momenta of $\bar{\Gamma}$ which are induced by the canonical momenta of $\Gamma$.

Another generalization concerns the number of substractions. Sometimes it is convenient to take more substractions than would actually be necessary for convergence. For self-energy diagrams, for instance, one will always take at least two substractions even if the diagram should be convergent. To include this possibility we introduce a function $d(\gamma)$ which assigns to every proper subdiagram $\gamma$ of $\Gamma$ an integer larger or equal to the dimension of $\gamma$

$$
d(\gamma) \geqq \sum_{a b \sigma} d\left(L_{a b \sigma}\right)+\sum_{a} d\left(V_{a}\right)+4 m(\gamma) .
$$

$d(\gamma)$ is called the degree of $\gamma^{\star}$. Proper diagrams of non-negative degree are called renormalization parts relative to $d(\gamma)$.

Relative to $d(\gamma)$ and an admissable set of basic internal momenta the finite part and the functions $R_{\gamma}, O_{\gamma}, \bar{R}_{\gamma}$ are then defined by the same equations $(2.41-2.44)$.

\section{Explicit Form of the Finite Part}

The integrand $R_{\Gamma}$ of the finite part was defined recursively by Eqs. (2.41-2.44). In this section we will derive explicit formulae for the function $R_{\Gamma}$. We begin with some combinatorial definitions concerning subdiagrams of a given diagram $\Gamma$. The diagrams $\gamma_{1}, \gamma_{2}$ are said to overlap

$$
\gamma_{1} \circ \gamma_{2}
$$

if none of the following three relations holds

$$
\gamma_{1} \cong \gamma_{2}, \gamma_{2} \cong \gamma_{1}, \gamma_{1} \cap \gamma_{2}=0 \text {. }
$$

Otherwise $\gamma_{1}, \gamma_{2}$ are called non-overlapping

$$
\gamma_{1} \curvearrowright \gamma_{2} \text {. }
$$

Let $\Gamma$ be any diagram. A $\Gamma$-forest $U$ is a set of diagrams satisfying the following conditions

$\star$ In addition we require $d(\gamma) \geqq d(\bar{\gamma})+\sum d\left(\gamma_{x}\right)$ for any reduced diagram $\bar{\gamma}=\gamma / \gamma_{1} \ldots \gamma_{c}$ with $d(\bar{\gamma})$ defined by $(2.38)$. 
(i) the elements of $U$ are proper subdiagrams of $\Gamma$,

(ii) any two elements $\gamma^{\prime}, \gamma^{\prime \prime}$ are non-overlapping

$$
\gamma^{\prime} \oslash \gamma^{\prime \prime},
$$

(iii) $U$ may also be the empty set.

If in addition each element of $U$ is a renormalization part we call $U$ a restricted $\Gamma$-forest.

Any subset $U^{\prime}$ of a $\Gamma$-forest $U$ is again a $\Gamma$-forest. All possible $\Gamma$ forests are partially ordered by $C$. A $\Gamma$-forest $U$ is called maximal if there is no other $\Gamma$-forest $U^{\prime}$ such that $U \subset U^{\prime}$. Let $U_{1}, \ldots, U_{c}$ be the maximal $\Gamma$-forests. Then all possible $\Gamma$-forests are given by the subsets of any $U_{a}$.

We will next be concerned with the structure of a given $I$-forest $U$. An element $\gamma$ of $U$ is called maximal (minimal) if there is no other $\gamma^{\prime} \in U$ such that $\gamma \subset \gamma^{\prime}$ or $\gamma^{\prime} \subset \gamma$ resp. Let $\gamma^{\prime}, \gamma^{\prime \prime}$ be two maximal elements of $U$. Since $\gamma^{\prime} C \gamma^{\prime \prime}$ and $\gamma^{\prime \prime} C \gamma^{\prime}$ are excluded we must have

$$
\gamma^{\prime} \cap \gamma^{\prime \prime}=0
$$

for maximal elements of $U$.

Let $\gamma$ be any diagram of $U$. Denote by $U(\gamma)$ the set of all $\gamma^{\prime} \in U$ satisfying $\gamma^{\prime} \subset \gamma . U(\gamma)$ is a $\Gamma$-forest as well as a $\gamma$-forest.

Let $\gamma_{1}, \ldots, \gamma_{c}$ be the maximal elements of $U(\gamma)$. Then we define

$$
\bar{\gamma}(U)=\gamma / \gamma_{1} \ldots \gamma_{c} .
$$

A $\Gamma$-forest $U$ containing $\Gamma$ itself is called full, a $\Gamma$-forest $U$ not containing $\Gamma$ is called normal. If $\Gamma$ is no renormalization part all restricted $\Gamma$ forests are normal. If $\Gamma$ is a renormalization part then there is a one-toone correspondence between full restricted $\Gamma$-forests $T$ and normal restricted $\Gamma$-forests $U$ given by

$$
T=U \cup\{\Gamma\} .
$$

Note that the empty set $U=0$ corresponds to $T=\{\Gamma\}$.

Let $U$ be a normal $\Gamma$-forest and $\gamma$ be an element of $U$. By $P(\gamma)$ we denote the set of all $\gamma^{\prime} \in U$ with

$$
\gamma^{\prime} \supseteqq \gamma .
$$

Since

$$
\gamma^{\prime} \cap \gamma^{\prime \prime} \neq \theta
$$

for any two elements of $P(\gamma)$ the set $P(\gamma)$ is totally ordered by C.

We now define the position $n(\gamma)$ of $\gamma$ in $U$ by the number of elements contained in $P(\gamma)$. Any two renormalization parts with the same position in $U$ are disjoint. 
Let $T$ be a full $\Gamma$-forest. Then we assign the position 0 to $\Gamma$ in $T$.

For any other element $\gamma \in T$ we define the position in $T$ by the position which $\gamma$ has in

$$
U=T \backslash\{\Gamma\} .
$$

Let $\mathscr{L}(U)$ denote the set of all lines which belong to at least one diagram of $U$. All elements of $U$ containing a given line $L_{a b \sigma} \in \mathscr{L}(U)$ are totally ordered by $C$. Hence there is a uniquely determined element $\gamma_{a b \sigma}$ with

$$
L_{a b \sigma} \in \mathscr{L}\left(\gamma_{a b \sigma}\right)
$$

such that

$$
L_{a b \sigma} \notin \mathscr{L}(\gamma) \quad \text { if } \quad \gamma \subset \gamma_{a b \sigma}, \gamma \in U
$$

Therefore $\mathscr{L}(U)$ is partitioned into mutually disjoint sets $\mathscr{L}(\bar{\gamma}(U))$

$$
\begin{gathered}
\mathscr{L}(U)=\bigcup_{\gamma \in U} \mathscr{L}(\bar{\gamma}(U)), \\
\bar{\gamma}(U) \cap \bar{\gamma}^{\prime}(U)=0 \text { for } \gamma \neq \gamma^{\prime} .
\end{gathered}
$$

The discussion of the recursive Eq. (2.43) can considerably be simplified by introducing substitution operators of the following kind. $S_{\mu}$ denotes the substitution operator

$$
S_{\mu}: K^{\gamma} \rightarrow K^{\gamma}\left(K^{\mu}\right), q^{\gamma} \rightarrow q^{\gamma}\left(K^{\mu}, q^{\mu}\right) \text { for } \quad \gamma \subset \mu .
$$

$S_{\Gamma}$ denotes the substitution operator

$$
S_{\Gamma}: K^{\gamma} \rightarrow K^{\gamma}(K), q^{\gamma} \rightarrow q^{\gamma}(K, q) .
$$

More precisely $S_{\mu}$ is defined as follows. Let $f$ be a function of the variables

$$
K, K^{\gamma}, q, q^{\gamma}
$$

where $\gamma$ runs over all renormalization parts of $\Gamma$. Then $S_{\mu} f$ denotes the function which is obtained from $f$ by substituting

$$
K_{\mu}^{\gamma}\left(K^{\mu}\right), q_{\mu}^{\gamma}\left(K^{\mu}, q^{\mu}\right)
$$

for all variable $K^{\gamma}, q^{\gamma}$ with $\gamma \subset \mu$. With the notation

$$
K^{\Gamma}=K, q^{\Gamma}=q
$$

this definition holds for $S_{\Gamma}$ too. In addition we use the abbreviation

$$
t^{\mu}=t_{q^{\mu}}^{d(\mu)} .
$$


With this notation the defining Eqs. (2.41) and (2.43) for $R_{\Gamma}$ and $O_{\gamma}$ become

$$
\begin{gathered}
R_{\Gamma}(K, q)=I_{\Gamma}(K q)+S_{\Gamma} \sum_{\gamma_{1} \ldots \gamma_{c}} I_{\Gamma / \gamma_{1} \ldots \gamma_{c}}(K q) \prod_{\tau=1}^{c} O_{\gamma_{\tau}}\left(K^{\gamma_{\tau}} q^{\gamma_{\tau}}\right), \\
O_{\gamma}\left(K^{\gamma} q^{\gamma}\right)=-t^{\gamma} I_{\gamma}\left(K^{\gamma} q^{\gamma}\right)-t^{\gamma} S_{\gamma} \sum_{\gamma_{1} \ldots \gamma_{c}}^{\prime} I_{\gamma / \gamma_{1} \ldots \gamma_{c}} \prod_{\tau=1}^{c} O_{\gamma_{\tau}}\left(K^{\gamma_{\tau}} q^{\gamma_{\tau}}\right) .
\end{gathered}
$$

The following lemma states an explicit formula for the $O$-functions.

Lemma 3.1. The $O$-function of a renormalization part $\gamma$ of $\Gamma$ may explicitly be written as

$$
O_{\gamma}\left(K^{\gamma} q^{\gamma}\right)=-t^{\gamma} S_{\gamma} \sum_{U \in \mathcal{N}_{r}} \prod_{\lambda \in U}\left(-t^{\lambda} S^{\lambda}\right) I_{\gamma}(U)
$$

or

$$
O_{\gamma}\left(K^{\gamma} q^{\gamma}\right)=\sum_{T \in \mathscr{F}_{r}} \prod_{\lambda \in T}\left(-t^{\lambda} S_{\lambda} I_{\gamma}(T)\right.
$$

The sum in (3.9) extends over the set $\mathcal{N}_{r}$ of all normal restricted $\gamma$-forests $U$ including the empty set. The sum (3.18) extends over the set $\mathscr{F}_{r}$ of all full restricted $\gamma$-forests. $I_{\gamma}(U)$ is essentially the function $I_{\gamma}$ but with a special choice of the variables. We define

$$
I_{\gamma}(U)=\prod_{a b \sigma} \Delta_{F}^{a b \sigma} \prod_{a} P_{a}
$$

with (2.5-2.6) and the substitutions

$$
l_{a b \sigma}=l_{a b \sigma}^{\gamma}\left(K^{\gamma} q^{\gamma}\right) \quad \text { if } \quad L_{a b \sigma} \in \mathscr{L}(\bar{\gamma})
$$

and

$$
l_{a b \sigma}=l_{a b \sigma}(K, q) \quad \text { if } \quad L_{a b \sigma} \notin \mathscr{L}(U) .
$$

In the product

$$
\prod_{\gamma \in U}\left(-t^{\gamma} S_{\gamma}\right)
$$

the factors $-t_{\gamma} S^{\gamma}$ are ordered from left to right according to increasing position. For elements of equal position in $U$ the order is irrelevant since

$$
t^{\gamma} S_{\gamma} t^{\gamma^{\prime}} S_{\gamma^{\prime}}=t^{\gamma^{\prime}} S_{\gamma^{\prime}} t^{\gamma} S_{\gamma} \quad \text { for } \quad \gamma \cap \gamma^{\prime}=0 .
$$

Proof. We use the notation

$$
D(U)=\prod_{\gamma \in U}\left(-t^{\gamma} S_{\gamma}\right) .
$$

First we note that the function

$$
\tilde{O}_{\gamma}(K q)=-t^{\gamma} S_{\gamma} \sum_{U \in \mathcal{N}} D(U) I_{\gamma}(U)
$$


may also be written as

$$
\tilde{O}_{\gamma}(K q)=\sum_{T \in \mathscr{F}} D(T) I_{\gamma}(T)
$$

using the relation (3.2) between full and normal forests. We prove $\tilde{O}_{\gamma}=O_{\gamma}$ by showing that $\tilde{O}_{\gamma}$ solves the recursive Eq. (2.39). First we rewrite the right hand side of (3.15)

$$
\sum_{U \in \mathcal{N}} D(U) I_{\gamma}(U)=-t^{\gamma} I_{\gamma}\left(K^{\gamma} q^{\gamma}\right)-t^{\gamma} S_{\gamma} \sum_{\gamma_{1} \ldots \gamma_{c}}^{\prime} \sum_{U \in K\left(\gamma_{1} \ldots \gamma_{c}\right)} D(U) I_{\gamma}(U) .
$$

Here $K\left(\gamma_{1} \ldots \gamma_{c}\right)$ is the class of all normal restricted $\gamma$-forests having the maximal elements $\gamma_{1}, \ldots, \gamma_{c}$. The first term of the r.h.s. of (3.17) corresponds to $U=\theta$. Now any $U \in K\left(\gamma_{1} \ldots \gamma_{c}\right)$ has the form

$$
U=T_{1}\left(\gamma_{1}\right) \cup \cdots \cup T_{c}\left(\gamma_{c}\right)
$$

where $T_{\tau}\left(\gamma_{\tau}\right)$ is the set of all $\gamma \in U$ with $\gamma \cong \gamma_{\tau} . T_{\tau}\left(\gamma_{\tau}\right)$ is a full restricted $\gamma_{\tau^{-}}$ forest. On the other hand any set $T_{1}, \ldots, T_{c}$ of full restricted $\gamma_{\tau}$-forests defines a $U \in K\left(\gamma_{1} \ldots \gamma_{c}\right)$ by

Hence

$$
U=T_{1} \cup \cdots \cup T_{c} .
$$

$$
\begin{aligned}
\sum_{U \in K\left(\gamma_{1} \ldots \gamma_{c}\right)} D(U) I_{\gamma}(U)=\prod_{\tau=1}^{c} & \sum_{T_{\tau} \in \mathscr{F}_{\tau}} D\left(T_{\tau}\right) I_{\gamma}(U) \\
& =I_{\gamma / \gamma_{1} \ldots \gamma_{c}}\left(K^{\gamma} q^{\gamma}\right) \prod_{\tau=1}^{c} \sum_{T_{\tau} \in \mathscr{F}_{\tau}} D\left(T_{\tau}\right) I_{\gamma_{\tau}}\left(T_{\tau_{\tau}}\right)
\end{aligned}
$$

where $\mathscr{F}_{\tau}$ denotes the set of all full $\gamma_{\tau}$-forests. Using $(3.16-3.17)$ we get

$$
\tilde{O}_{\gamma}\left(K^{\gamma}, q^{\gamma}\right)=-t^{\gamma} I_{\gamma}\left(K^{\gamma}, q^{\gamma}\right)-t^{\gamma} S_{\gamma} \sum_{\gamma_{1} \ldots \gamma_{c}}^{\prime \gamma} I_{\gamma / \gamma_{1} \ldots \gamma_{c}}\left(K^{\gamma}, q^{\gamma}\right) \prod_{\tau=1}^{c} \tilde{O}_{\gamma_{\tau}}\left(K^{\gamma_{\tau}}, q^{\gamma_{\tau}}\right)
$$

which proves $\tilde{O}_{\gamma}=O_{\gamma}$.

Theorem 3.1. The function $R_{\Gamma}$ is given explicitly by

$$
R_{\Gamma}(K q)=S_{\Gamma} \sum_{U \in \mathscr{U}_{r}} \prod_{\gamma \in U}\left(-t^{\gamma} S_{\gamma}\right) I_{\Gamma}(U)
$$

with the sum extending over the set $\mathscr{U}_{r}$ of all restricted $\Gamma$-forests.

Proof. From (3.7) and the explicit formula (3.10) for $O_{\gamma}$ we obtain

$$
\begin{aligned}
R_{\Gamma}(K q) & =I_{\Gamma}(K q)+S_{\Gamma} \sum_{\gamma_{1} \ldots \gamma_{c}} I_{\Gamma / \gamma_{1} \ldots \gamma_{c}}(K q) \prod_{\tau=1}^{c} \sum_{T_{\gamma_{\tau}} \in \mathscr{F}_{\tau}} D\left(T_{\gamma_{\tau}}\right) I_{\gamma_{\tau}}\left(T_{\gamma_{\tau}}\right) \\
& =I_{\Gamma}(K q)+S_{\Gamma} \sum_{\gamma_{1} \ldots \gamma_{c}} \sum_{U \in K\left(\gamma_{1} \ldots \gamma_{c}\right)} D(U) I_{\Gamma}(U) .
\end{aligned}
$$


Formula (3.21) can considerably be simplified by using the identity

$$
\prod_{\gamma \in U_{0}}\left(1-t^{\gamma}\right) S_{\gamma} I_{\Gamma}\left(U_{0}\right)=\sum_{U \subseteq U_{0}} \prod_{\gamma \in U}\left(-t^{\gamma} S_{\gamma}\right) I_{\gamma}(U)
$$

which holds for any $\Gamma$-forest $U_{0}$. Let now $\Gamma$ be a diagram with no overlapping divergencies, i.e.

$$
\gamma_{1} \otimes \gamma_{2}
$$

for any two renormalization parts of $\Gamma$. Then the set $U$ of all renormalization parts is a $\Gamma$-forest. The subsets of $U_{0}$ form all possible restricted $\Gamma$-forests. Using (3.21) and (3.22) we obtain the following theorem.

Theorem 3.2. Let $\Gamma$ be a Feynman diagram with no overlapping renormalization parts. Then the integral of the finite part is given by

$$
R_{\Gamma}(K q)=S_{\Gamma} \prod_{\gamma \in U_{0}}\left(1-t^{\gamma}\right) S_{\gamma} I_{\Gamma}\left(U_{0}\right)
$$

where the product extends over all renormalization parts of $\Gamma$.

Formula (3.23) represents Dyson's prescription for removing nonoverlapping divergencies [16]. Using the power counting theorem it is not difficult to prove that the corresponding integral (2.19) is absolutely convergent. A generalization of (3.23) to the case of overlapping divergencies can be given. The formula obtained, however, is not useful for proving convergence. We therefore quote the result only.

Theorem 3.3. Let $U_{1}, \ldots, U_{c}$ be the maximal restricted $\Gamma$-forests of a diagram $\Gamma$. Form the intersections

$$
U_{i_{1} \ldots i_{v}}=U_{i_{1}} \cap \cdots \cap U_{i_{v}}
$$

for all subsets

$$
\left(i_{1} \ldots i_{v}\right) \cong(1, \ldots, c)
$$

( some of the intersection (3.24) may be empty). The integrand of the finite part is then given by

$$
R_{\Gamma}(K q)=S_{\Gamma} \sum_{i_{1} \ldots i_{v}}(-1)^{v+1} \prod_{\gamma \in U_{i_{1}} \ldots U_{l_{v}}}\left(1-t^{\gamma}\right) S_{\gamma} I_{\Gamma}\left(U_{i_{1} \ldots i_{v}}\right) .
$$

For the convergence proof of the following section it is convenient to use Eq. (3.21) in a more general form given by the following theorem.

Theorem 3.4. The function $R_{\Gamma}$ is given explicitly by

$$
R_{\Gamma}(K q)=S_{\Gamma} \sum_{U \in \mathscr{U}} \prod_{\gamma \in U}\left(-t^{\gamma} S_{\gamma}\right) I_{\Gamma}(U)
$$


with the sum extending over the set $U$ of all $\Gamma$-forests. Here the convention is used that

$$
t^{\gamma}=0 \text { of } \quad d(\gamma)<0 .
$$

The proof is trivial since on account of (3.27) all non-restricted $\Gamma$ forests give zero contribution to (3.26).

\section{Convergence Proof}

In this section it will be shown that the finite part

$$
\int R_{\Gamma}(K(k), q) d k_{1} \ldots d k_{m}
$$

satisfies the requirements of the power counting theorem. In a previous paper the power counting theorem was proved for integrals of the form

$$
\int d k \frac{P(k, q)}{\prod_{j=1}^{m}\left(l_{j 0}^{2}-l_{j}^{2}-\mu_{j}^{2}+i \varepsilon\left(\boldsymbol{l}_{j}^{2}+\mu_{j}^{2}\right)\right.}
$$

where $P(k, q)$ is a polynomial in $k$ and $q$. Clearly (4.1) is of the form (4.2) since $R_{\Gamma}(K, q)$ may be written as

$$
\begin{aligned}
& R_{\Gamma}=\frac{A}{B_{1} B_{2}}, \\
& B_{1}=\prod_{a b \sigma}\left(l_{a b \sigma}^{2}-\mu_{a b \sigma}^{2}+i \varepsilon\left(\boldsymbol{l}_{a b \sigma}^{2}+\mu_{a b \sigma}^{2}\right)\right), \\
& B_{2}=\prod_{\gamma} \prod_{a b \sigma}\left\{k_{a b \sigma}^{\gamma 2}-\mu_{a b \sigma}^{2}+i \varepsilon\left(\boldsymbol{k}_{a b \sigma}^{\gamma}+\mu_{a b \sigma}^{2}\right)\right\}^{c(\gamma a b \sigma)}, \\
& l_{a b \sigma}=k_{a b \sigma}+q_{a b \sigma}(q), \quad k_{a b \sigma}^{\gamma}=k_{a b \sigma}^{\gamma}(K)
\end{aligned}
$$

where $A$ is a polynomial in $K$ and $q$. The product $\prod_{\gamma}$ extends over all renormalization parts $\gamma$ of $\Gamma$.

The hypothesis of the power counting theorem is contained in the following theorem

Theorem 4.1. The finite part of a Feynman integral

$$
\int_{H} d k R_{\Gamma}(K(k), q)
$$

has negative dimension for $R_{4 m}$ and any hyperplane $H$ described by a set of linear equations

$$
\begin{aligned}
k & =k(t)=a+b t, \\
a & =\left(a_{i}\right), t=\left(t_{j}\right), b=\left(b_{i j}\right), \\
i & =1, \ldots, m ; j=1, \ldots, h .
\end{aligned}
$$


With this result the power counting theorem (Theorem 2 of Ref. [6]) implies that (4.1) is absolutely convergent for $\varepsilon>0$ and approaches a well defined distribution in the limit $\varepsilon \rightarrow+0$.

For the proof of the theorem we begin with a couple of definitions. A $\Gamma$-forest $U$ is called complete on $H$ if $\Gamma \in U$ and if for any $\gamma \in U$

either (i) all lines $L_{a b \sigma} \in \mathscr{L}(\bar{\gamma})$ are variable on $H$ relative to $\gamma$

or (ii) all lines $L_{a b \sigma} \in \mathscr{L}(\bar{\gamma})$ are constant on $H$ relative to $\gamma$. A line $L_{a b \sigma} \in \mathscr{L}(\Gamma)$ is called constant on $H$ relative to $\gamma$ if

$$
k_{a b \sigma}^{\gamma}=\text { const on } H \text {, i.e. } k_{a b \sigma}^{\gamma}(T)=\text { const. }
$$

Let $U$ be an arbitrary forest of $\Gamma$. We are going to define a completion $\bar{U}$ of $U$ which will be shown to be the unique minimal complete forest containing $U$.

We begin defining $\bar{U}$ for a full $U$, i.e. $\Gamma \in U$. Let $W(U)$ be the set of all $\gamma \in U$ with the property that at least one line of $\bar{\gamma}(U)$ is constant relative to $\gamma$. For any $\gamma \in W(U)$ let $s(\gamma U)$ be the subdiagram of $\gamma$ which is spanned by the set of constant lines of $\bar{\gamma}(U)$ relative to $\gamma^{5}$. Let $\delta_{1}, \ldots, \delta_{c}$ be the connected components of $\gamma \backslash s(\gamma U)^{3}$. We first show that each $\delta_{a}$ is proper.

Lemma 4.1. Each connected component of $\gamma \backslash s(\gamma U)$ is proper.

Proof. Assume that $L_{a b \sigma} \in \mathscr{L}(\gamma)$ is an improper line of $\gamma \backslash s(\gamma U)$. Then momentum conservation at each vertex implies

$$
k_{a b \sigma}^{\gamma}=\sum c_{i} k_{a_{1} b_{i} \sigma_{i}}^{\gamma}, \quad L_{a_{i} b_{1} \sigma_{l}} \in \mathscr{L}(s(\gamma U)) .
$$

By definition of $s(\gamma U)$ the momenta $k_{a_{i} b_{i} \sigma_{i}}^{\gamma}$ are constant on $H$, hence also $k_{a b \sigma}^{\gamma}$ is constant on $H$. If $L_{a b \sigma} \in \mathscr{L}(\bar{\gamma})$ we have a contradiction because $s(\gamma U)$ is the set of all constant lines of $\bar{\gamma}(U)$. Therefore $L \notin \mathscr{L}(\bar{\gamma})$, i.e. $L \in \mathscr{L}(\varphi)$ with $\varphi \in U, \varphi \subset \gamma$. Since $\varphi$ is connected we have $\varphi \leqq \delta_{a}$. If $L$ were an improper line of $\delta_{a}$ it would also be an improper line of $\varphi$ which is impossible. This completes the proof that $\gamma \backslash s(\gamma U)$ does not contain improper lines i.e. each $\delta_{a}$ must be proper.

We define $\mathscr{A}(U)$ as the set of all diagrams $\tau \notin U$ which are connected components $\delta_{a}$ of $\gamma \backslash s(\gamma U)$ with $\gamma \in W(U)$. The completion of a full forest $U$ is then defined by

$$
\bar{U}=U \cup \mathscr{A}(U) \text {. }
$$

Our first aim is to show that $\bar{U}$ is a forest. We begin with

Lemma 4.2. If $\tau$ is a connected component of $\gamma \backslash s(\gamma U)$ and $\gamma^{\prime} \in U$, $\gamma^{\prime} \subset \gamma$ we have

$$
\gamma^{\prime} \cap \tau=0 \quad \text { or } \quad \gamma^{\prime} \leqq \tau .
$$

${ }^{5}$ Note that $s(\gamma(U))$ is defined as a subdiagram of $\gamma$ but not of $\bar{\gamma}(U)$. That means that no vertices of are identified in $s(\gamma(U))$. 
Proof. Since all $\gamma^{\prime} \subset \gamma, \gamma^{\prime} \in U$ are connected and $\gamma^{\prime} \leqq \gamma \backslash s(\gamma U)$ it follows $\gamma^{\prime} \leqq \delta_{a}$ where $\delta_{a}$ is a connected component of $\gamma \backslash s(\gamma U)$. Hence $\gamma^{\prime} \leqq \delta_{a} \neq \tau$ or $\gamma^{\prime} \leqq \tau$. In the first case $\gamma^{\prime} \cap \tau=0$, in the second case $\gamma^{\prime} \leqq \tau$.

Lemma 4.3. $U$ is a forest.

Proof. (i) We first prove that any elements $\tau \in \mathscr{A}(U)$ and $\gamma^{\prime} \in U$ do not overlap. Let $\tau$ be a connected component of $\gamma \backslash s(\gamma U)$. We have

$$
\gamma^{\prime} \supset \gamma, \gamma^{\prime} \cap \gamma=0 \text { or } \gamma^{\prime} \subset \gamma \text {. }
$$

$\gamma^{\prime} \supset \gamma$ implies $\gamma^{\prime} \supset \tau . \gamma^{\prime} \cap \gamma=\theta$ implies $\gamma^{\prime} \cap \tau=\theta$. $\gamma^{\prime} \subset \gamma$ implies $\gamma^{\prime} \subset \tau$ or $\gamma^{\prime} \cap \tau=\theta$ (Lemma 4.2). Hence $\gamma^{\prime} \oslash \tau$ for any $\tau \in \mathscr{A}(U)$ and $\gamma^{\prime} \in U$.

(ii) We next show that any two different elements $\tau_{1}, \tau_{2} \in \mathscr{A}$ do not overlap.

(a) Let $\tau_{1}, \tau_{2}$ both be connected components of $\gamma \backslash s(\gamma U)$. Then $\tau_{1} \cap \tau_{2}=\theta$.

(b) Let $\tau_{1}$ be a connected component of $\gamma_{1} \backslash s\left(\gamma_{1} U\right), \tau_{2}$ be a connected component of $\gamma_{2} \backslash s\left(\gamma_{2} U\right)$. If $\gamma_{1} \subset \gamma_{2}$ Lemma 4.2 implies $\gamma_{1} \subset \gamma_{2}$ or $\tau_{2} \cap \tau_{2}=0$. Hence $\tau_{1} \subset \tau_{2}$ or $\tau_{1} \cap \tau_{2}=\theta$.

If on the other hand $\gamma_{1} \cap \gamma_{2}=\theta$ then also $\tau_{1} \cap \tau_{2}=\theta$. This completes the proof that $U$ is a forest.

Lemma 4.4. Let $\tau \in \mathscr{A}(U)$ be a connected component of $\gamma \backslash s(\gamma U)$. Let $U(\tau)$ be the set of all $\sigma \in U$ with $\sigma \subset \tau$ and $\gamma_{1}, \ldots, \gamma_{c}$ be the maximal element of $U(\tau)$. Then all lines of

$$
\bar{\tau}(\bar{U})=\tau / \gamma_{1} \ldots \gamma_{c}
$$

are variable relative to $\tau$.

Proof. Let $L_{a b \sigma} \in \mathscr{L}(\bar{\tau}(\bar{U}))$. By definition

$$
\begin{gathered}
k_{a b \sigma}^{\tau}+q_{a b \sigma}^{\tau}\left(q^{\tau}\right)=k_{a b \sigma}^{\gamma}+q_{a b \sigma}^{\gamma}\left(q^{\gamma}\right), \\
q^{\tau}=q^{\tau}\left(K^{\gamma} q^{\gamma}\right), k_{a b \sigma}^{\tau}=k_{a b \sigma}^{\tau}\left(K^{\gamma}\right) .
\end{gathered}
$$

Setting $q^{\gamma}=0$ we obtain

$$
k_{a b \sigma}^{\tau}+q_{a b \sigma}^{\tau}\left(q^{\tau}\right)=k_{a b \sigma}^{\gamma}, q^{\tau}=q^{\tau}\left(K^{\gamma}, 0\right), k_{a b \sigma}^{\tau}=k_{a b \sigma}^{\tau}\left(K^{\gamma}\right) .
$$

Since $\tau$ is a connected component of $\gamma \backslash s(\gamma U)$ we have

$$
q_{a}^{\tau}\left(K^{\gamma}, 0\right)=\sum c_{i} k_{a_{i} b_{l} \sigma_{l}}^{\gamma}, \quad L_{a_{l} b_{i} \sigma_{i}} \in \mathscr{L}(s(\gamma U)) .
$$

Hence all $q_{a}^{\tau}\left(K^{\gamma}, 0\right)$ are constant on $H$. If $k_{a b \sigma}^{\tau}$ is constant on $H$ Eq. (4.8) implies that $k_{a b \sigma}^{\gamma}$ is also constant on $H$ in contradiction to $L_{a b \sigma} \in \mathscr{L}(\bar{\tau})$ $\subseteq \mathscr{L}(\bar{\gamma} \backslash s(\gamma U))$. 
Lemma 4.5. $U$ is complete.

Proof. (i) $\Gamma \in U$.

(ii) Let $\gamma \in U, \gamma \notin W(U)$. All lines of $\bar{\gamma}(\bar{U})$ are variable relative to $\gamma$ since $\bar{\gamma}(\bar{U})=\bar{\gamma}(U)$.

(iii) Let $\gamma \in W(U)$. Then $\mathscr{L}(\bar{\gamma}(\bar{U}))=\mathscr{L}(s(\gamma U))$ i.e. all lines of $\bar{\gamma}(U)$ are constant relative to $\gamma$.

(iv) Let $\tau \in \mathscr{A}(U)$. Then $\tau(\bar{U})=\tau / \gamma_{1} \ldots \gamma_{c}$ where $\gamma_{1} \ldots \gamma_{c}$ are the maximal elements of $U(\tau)$. Lemma 4.4 implies that all lines of $\bar{\tau}(\bar{U})$ are variable relative to $\tau$.

Lemma 4.6. Let $U$ be a full forest. Any set $V$ with

$$
U \subseteq V \cong \bar{U}
$$

is a forest with completion $\bar{U}$.

Proof. Clearly $V$ is a forest. We will show that $W(V)=W(U)$.

(i) $\gamma \in W(U)$ implies $\gamma \in W(V)$ since $s(\gamma U)=s(\gamma V)$.

(ii) $\gamma \in U, \gamma \in W(V)$ implies $\gamma \in W(U)$ since

$$
\mathscr{L}(\bar{\gamma}(V)) \cong \mathscr{L}(\bar{\gamma}(U)) .
$$

(iii) If $\tau \in V \backslash U \subseteq \mathscr{A}(U)$ all lines of $\bar{\tau}(V)=\bar{\tau}(U)$ are variable.

Combining (i)-(iii) we obtain $W(V)=W(U)$. Hence

$$
\mathscr{A}(V)=\mathscr{A}(U) \backslash(V \backslash U)
$$

and $\bar{V}=\bar{U}$.

Next we will define the base $\underline{U}$ of a forest $U$ which will turn out to be the minimal forest among all forests with the completion $\bar{U}$. The set $\mathscr{B}(U)$ is defined as the set of all diagrams $\tau \in U$ satisfying

(i) $\tau \notin W(U)$.

(ii) $\tau$ is a connected component of $\gamma \backslash s(\gamma U)$ with $\gamma \in W(U)$. Let $U$ be full. The base $\underline{U}$ of $U$ is defined by

$$
\underline{U}=U \backslash \mathscr{B}(U) \backslash\{\Gamma\}
$$

$\underline{U}$ is a forest. Furthermore define a full forest $\underline{U}^{\prime}$ by

$$
\underline{U}^{\prime}=U \backslash \mathscr{B}(U) \text {. }
$$

Lemma 4.7. If $U$ is a complete forest on $H$ the sets $W(U)$ and $\mathscr{B}(U)$ are given by the following conditions:

$W(U)$ is the set of all $\gamma \in U$ for which all lines of $\bar{\gamma}(U)$ are constant relative to $\gamma$.

If $\gamma \notin W(U)$ all lines of $\bar{\gamma}(U)$ are variable on $H$ relative to $\gamma$.

$\mathscr{B}(U)$ is the set of all diagrams $\tau \in U$ satisfying

(i) $\tau \notin W(U)$.

(ii) $\tau$ is a maximal element of $U(\gamma)$ with $\gamma \in W(U)$. 
Proof. The first and the second statement follow immediately from the definition of $W(U)$.

If $U$ is complete we have

$$
\mathscr{L}(s(\gamma U))=\mathscr{L}(\bar{\gamma}(U)) \quad \text { for } \quad \gamma \in W(U)
$$

or

$$
\mathscr{L}(\gamma \backslash s(\gamma U))=\mathscr{L}\left(\gamma_{1} \cup \cdots \cup \gamma_{c}\right),
$$

where $\gamma_{1} \ldots \gamma_{c}$ are the maximal elements of $U(\gamma)$. Hence the connected components of $\gamma \backslash s(\gamma U)$ are identical with the maximal elements $\gamma_{a}$ of $U(\gamma)$. This proves the last statement.

Lemma 4.8. For any full forest $U$ holds

$$
W\left(\underline{U}^{\prime}\right)=W(U) .
$$

Proof. (i) If $\tau \in U \backslash \underline{U}^{\prime}=\mathscr{B}(U)$ the diagram $\bar{\tau}(U)$ does not contain any constant lines relative to $\gamma$. Hence

$$
\tau \in U \backslash \underline{U}^{\prime} \text { implies } \tau \notin W(U) .
$$

(ii) Let $\gamma \in \underline{U}^{\prime}, \gamma \in W(U)$. Then $\bar{\gamma}(U)$ contains a line $L_{a b \sigma}$ which is constant relative to $\gamma$. Since $\mathscr{L}(\bar{\gamma}(U)) \cong \mathscr{L}\left(\bar{\gamma}\left(\underline{U}^{\prime}\right)\right)$ the line $L_{a b \sigma}$ belongs to $\bar{\gamma}\left(\underline{U}^{\prime}\right)$. Hence

$$
\gamma \in \underline{U}^{\prime}, \gamma \in W(U) \text { implies } \gamma \in W\left(\underline{U}^{\prime}\right) \text {. }
$$

(iii) Let $\gamma \in \underline{U}^{\prime}, \gamma \notin W(U)$. Then $\bar{\gamma}(U)$ does not contain a line which is constant relative to $\gamma$. By definition $\bar{\gamma}(U)=\gamma / \gamma_{1} \ldots \gamma_{c}$ where $\gamma_{1} \ldots \gamma_{c}$ are the maximal elements of $U(\gamma)$. Since $\gamma \notin W(U)$ each $\gamma_{a}$ belongs to $\underline{U}^{\prime}$, therefore $\bar{\gamma}\left(\underline{U}^{\prime}\right)=\bar{\gamma}(U)$. Hence

$$
\gamma \in \underline{U}^{\prime}, \gamma \notin W(U) \text { implies } \gamma \notin W\left(\underline{U}^{\prime}\right) .
$$

Combining (4.16-4.18) we obtain the statement of the lemma.

Lemma 4.9. For each $\gamma \in W\left(U^{\prime}\right)=W(U)$ the two sets $s(\gamma U), s\left(\gamma \underline{U}^{\prime}\right)$ are equal

$$
s(\gamma U)=s\left(\gamma \underline{U}^{\prime}\right)
$$

Proof. Let $\gamma \in W(U)$. Any line of $\bar{\gamma}(U)$ also belongs to $\bar{\gamma}\left(\underline{U}^{\prime}\right)$. Hence

$$
s(\gamma U) \cong s\left(\gamma \underline{U}^{\prime}\right) .
$$

Suppose that $L_{a b \sigma} \in \mathscr{L}\left(s\left(\gamma \underline{U}^{\prime}\right)\right) \backslash s(\gamma U) . L_{a b \sigma}$ must belong to a $\tau \in \mathscr{B}(U)$ which is a connected component of $\gamma \backslash s(\gamma U)$ with $\gamma \in W(U)$. It cannot belong to any $\sigma \in U$ with $\sigma \subset \tau$ since $\mathscr{L}\left(s\left(\gamma \underline{U}^{\prime}\right)\right) \subseteq \mathscr{L}\left(\bar{\gamma}\left(\underline{U}^{\prime}\right)\right)$. Hence $L_{a b \sigma} \in \bar{\tau}(U)$. Using Eqs. (4.7-4.9) of Lemma 4.4 we find that

$$
k_{a b \sigma}^{\gamma}=\text { const on } H \text { implies } k_{a b \sigma}^{\tau}=\text { const on } H
$$

in contradiction to the requirement on the elements of $\mathscr{B}$. 
We now extend the definition of completion and base to normal forests. If $U$ is a normal forest we define the completion $\bar{U}$ and the base $\underline{U}$ by

$$
\bar{U}=\bar{V}, \underline{U}=\underline{V} \text { where } \quad V=U \cup\{\Gamma\} .
$$

Lemma 4.10. Let $U$ be a given forest with completion $\bar{U}$ and base $\underline{U}$. $\bar{U}$ is the completion of $\underline{U}$ and $\underline{U}$ is the base of $\bar{U}$.

Proof. It is sufficient to consider a full forest $U$. Lemma 4.8 and 4.9 imply

$$
\mathscr{A}\left(\underline{U}^{\prime}\right)=\mathscr{B}(U) \cup \mathscr{A}(U) .
$$

With the notation $B=\underline{U}^{\prime}, C=\bar{U}$

$$
\bar{B}=B \cup \mathscr{B}(U) \cup \mathscr{A}(U)=U \cup \mathscr{A}(U)=C .
$$

Lemma 4.4 implies

$$
\mathscr{B}(C)=\mathscr{A}(U) \cup \mathscr{B}(U) .
$$

Hence $\underline{C}=C-\mathscr{B}(C)=B$.

Theorem 4.2. Let $C$ be a given complete forest with base $B$. The set of all forests $U$ with the completion $C$ is given by the condition

$$
B \subseteq U \subseteq C .
$$

Proof. Let $U$ have the completion $C$. Then $U \subseteq C$ and $\underline{U}=B$ (Lemma 4.10). Hence $B \leqq U \leqq C$.

Since $C$ is the completion of $B$ (Lemma 4.10) any $U$ satisfying (4.21) has the completion $C$.

Theorem 4.3. The finite part of the integrand of a Feynman integral is given by

$$
\begin{gathered}
R_{\Gamma}(K q)=S_{\Gamma} \sum_{U \in \mathscr{C}} X_{U}, \\
X_{U}=\prod_{\gamma \in U}\left(t(\gamma) S_{\gamma}\right) I_{\gamma}(U), \\
f(\gamma)=1-t^{\gamma} \quad \text { if } \quad \gamma \in \mathscr{B}(U), f(\gamma)=-t^{\gamma} \quad \text { if } \quad \gamma \notin \mathscr{B}(U) .
\end{gathered}
$$

The sum in (4.22) extends over the set $\mathscr{C}$ of all complete forests of $\Gamma$. The set $\mathscr{B}(U)$ is given by Lemma 4.7 .

Proof. Two forests are called equivalent if they have the same completion. According to the Theorem 4.2 the corresponding equivalence classes are given by the condition $C \subseteq U \leqq C$ where $C$ is a complete forest with base $C$. This partition of the set of all forests into equivalence 
classes leads to the following formula (see Eq. (3.21))

$$
R_{\Gamma}=\sum_{C \in \mathscr{C}} X_{C}, X_{C}=S_{\Gamma} \sum_{\underline{C} \subseteq U \subseteq C} \prod_{\gamma \in U}\left(-t^{\gamma} S_{\gamma}\right) I_{\Gamma}(U) .
$$

An alternative formula for $X_{C}$ is

$$
X_{C}=S_{\Gamma} \prod_{\gamma \in C}\left(f(\gamma) S_{\gamma}\right) I_{\Gamma}(C) .
$$

An equivalent definition of $f(\gamma)$ is

$$
\begin{array}{lll}
f(\gamma)=1-t^{\gamma} & \text { for } & \gamma \in C-\underline{C}=\mathscr{B}(C), \\
f(\gamma)=-t^{\gamma} & \text { for } & \gamma \in \underline{C} .
\end{array}
$$

In order to show the equivalence of the two formula for $X_{C}$ we work out the products of factors $\left(1-t^{\gamma}\right)$ in $(4.26)$

$$
\begin{gathered}
X_{C}=S_{\Gamma} \sum_{Q \subseteq \mathcal{C}-C} \prod_{\gamma \in Q}\left(g_{Q}(\gamma) S_{\gamma}\right) I_{\Gamma}(C), \\
g_{Q}(\gamma)=-t^{\gamma} \quad \text { for } \quad \gamma \in Q, \quad g_{Q}(\gamma)=1 \text { for } \gamma \notin Q .
\end{gathered}
$$

The sum extends over all subsets $Q$ of $C-\underline{C}$. Introducing $V=\underline{C}+Q$ as new variable of summation we obtain (4.26) which can be rewritten in the form (4.23).

The next aim is to give upper bounds for the degree of the function $X_{U}$ with respect to the parameters $T$ of the hyperplane $H$. This will eventually lead to the desired result that the dimension of the renormalized Feynman integral is always negative.

We first state a recursion formula determining $X_{U}$ which follows easily from the definition (4.23). The subscript $U$ will be omitted in the work that follows. For the sets $W(U), \mathscr{B}(U)$ given by Lemma 4.7 we will use the notation

$$
\mathscr{B}=\mathscr{B}(U), \quad W=W(U) .
$$

Lemma 4.11. For a given complete forest $U$ the function $X_{U}$ is determined by

$$
X_{U}=X=\left(1-t^{\Gamma}\right) Y_{\Gamma}
$$

where for any $y \in U$

$$
Y_{\gamma}=I_{\gamma / \gamma_{1} \ldots \gamma_{c}} S_{\gamma} f_{\gamma_{1}} Y_{\gamma_{1}} \ldots f_{\gamma_{c}} Y_{\gamma_{c}} .
$$

$\gamma_{1}, \ldots, \gamma_{c}$ denote the maximal elements of $U(\gamma)$. If $\gamma$ is minimal we set $Y_{\gamma}=I_{\gamma}$. $f_{\gamma}$ is defined by (4.24).

The function $Y_{\gamma}$ has the general form

$$
Y_{\gamma}\left(K^{\gamma} q^{\gamma}\right)=\frac{A}{B_{1} B_{2}}
$$


where $A$ is a polynomial in $K^{\gamma}, q^{\gamma}$ and

$$
\begin{gathered}
B_{1}=\prod_{a b \sigma}\left(l_{a b \sigma}^{2}-\mu_{a b \sigma}^{2}+i \varepsilon\left(\boldsymbol{l}_{a b \sigma}^{2}+\mu_{a b \sigma}^{2}\right)\right), \\
B_{2}=\prod_{\varphi \in U(\gamma)} \prod_{a b \sigma}\left(k_{a b \sigma}^{\varphi 2}-\mu_{a b \sigma}^{2}+i \varepsilon\left(\boldsymbol{k}_{a b \sigma}^{\varphi 2}+\mu_{a b \sigma}^{2}\right)\right)^{c(\gamma a b \sigma)}, \\
l_{a b \sigma}=k_{a b \sigma}^{\gamma}+q_{a b \sigma}^{\gamma}, \\
k_{a b \sigma}^{\varphi}=k_{a b \sigma}^{\varphi}\left(k^{\gamma}\right) .
\end{gathered}
$$

We next want to determine the degree of the function $Y_{\gamma}$. To this end the following lemma will be useful.

Lemma 4.12. Let $F$ be a function of the form

$$
Y=\frac{A}{C}, \quad C=\prod_{\alpha}\left(l_{\alpha}^{2}-\mu_{\alpha}^{2}+i \varepsilon\left(l_{\alpha}^{2}+\mu_{\alpha}^{2}\right)\right)
$$

where $A$ is a polynomial in $t_{1}, \ldots, t_{\varrho}$ and

$$
l_{\alpha}=a_{\alpha}+\sum c_{\alpha \beta} t_{\beta}+\sum d_{\alpha \beta} q_{\beta}
$$

with all

$$
\sum c_{\alpha \beta} t_{\beta} \neq 0
$$

Then the relation

implies

$$
\operatorname{degr}_{t, q} F \leqq l
$$

$$
\operatorname{degr}_{t}\left(1-t_{q}^{d}\right) F \leqq l-d-1
$$

$\operatorname{degr}_{x} F$ denotes the degree of a rational function $F$ with respect to the variables $x=\left(x_{1} \ldots x_{n}\right)$.

Proof. We decompose the polynomial

$$
A=\sum_{\gamma=0}^{g} A_{\gamma}
$$

such that $A_{\gamma}$ is homogeneous in $q$ of degree $\gamma$. (4.29) implies

if

$$
\operatorname{degre}_{t, q} A \leqq e+\delta
$$

$$
\operatorname{degr}_{t, q} C=\delta \text {. }
$$

From (4.35) we get

$$
\operatorname{degr}_{t} A_{\gamma} \leqq e+\delta-\gamma .
$$

Since $A_{\gamma}$ is homogeneous in $q$

$$
\left(1-t_{q}^{d}\right) \frac{A_{\gamma}}{C}=A_{\gamma}\left(1-t_{p}^{d-\gamma}\right) \frac{1}{C} .
$$


Here

$$
\operatorname{degr}\left(1-t_{q}^{d-\gamma}\right) \frac{1}{C} \leqq-\delta-d+\gamma-1
$$

Hence

$$
\begin{aligned}
\operatorname{degr}_{t}\left(1-t_{q}^{d}\right) \frac{A_{\gamma}}{C} & \leqq \operatorname{degr}_{t} A_{\gamma}+\operatorname{degr}_{t}\left(1-t_{q}^{d-\gamma}\right) \frac{1}{C} \\
& \leqq e-d-1
\end{aligned}
$$

using (4.34) and (4.36).

It is convenient to introduce the following integer

$$
M(\gamma)=4 \sum_{\mu}^{\gamma} m(\bar{\mu})
$$

where the sum extends over all $\mu$ satisfying the condition

$$
\mu \in U, \mu \subseteq \gamma \text { and } \mu \notin W \text {. }
$$

$m(\bar{\mu})$ is the number of independent internal momenta of $\bar{\mu}$. Apparently $M(\Gamma)$ is the number of independent integration variables on $H$. For $\bar{\mu}$ contains only variable lines if $\mu \notin W$ while $\bar{\mu}$ contains only constant lines if $\mu \in W$.

Lemma 4.13. The following inequalities hold

$$
\begin{gathered}
\operatorname{degr}_{t, q}^{\gamma} \quad Y_{\gamma}\left(K^{\gamma}(K), q^{\gamma}\right) \leqq d(\gamma)-M(\gamma) \quad \text { for } \quad \gamma \in U, \gamma \notin W, \\
\operatorname{degr}_{t} Y_{\gamma}\left(K^{\gamma}(K), q^{\gamma}\right)<-M(\gamma) \quad \text { for } \quad \gamma \in W .
\end{gathered}
$$

Proof. As hypothesis of induction we assume the inequalities to be valid for all maximal elements $\gamma_{a}$ of $U(\gamma)$. It will then be shown that the inequalities also hold for $\gamma$ itself.

(i) Case $\gamma \in W$. Then the recursion formula holds with

$$
\begin{gathered}
f_{\gamma_{\alpha}}=-t^{\gamma_{\alpha}} \quad \text { for } \quad \gamma_{\alpha} \in W . \\
f_{\gamma_{\alpha}}=1-t^{\gamma_{\alpha}} \text { for } \quad \gamma_{\alpha} \notin W .
\end{gathered}
$$

We will find the following relations

$$
\begin{gathered}
\operatorname{degr}_{t} I_{\gamma / \gamma_{1} \ldots \gamma_{c}}=0, \\
\operatorname{degr}_{t} S_{\gamma} t^{\gamma_{\alpha}} Y_{\gamma_{\alpha}}<-M\left(\gamma_{\alpha}\right) \quad \text { if } \quad \gamma_{\alpha} \in W, \\
\operatorname{degr}_{t} S_{\gamma}\left(1-t^{\gamma_{\alpha}}\right) Y_{\gamma_{\alpha}}<-M\left(\gamma_{\alpha}\right) \text { if } \gamma_{\alpha} \notin W
\end{gathered}
$$

(4.45-4.47) imply the inequality (4.44)

$$
\operatorname{degr}_{t} Y_{\gamma} \leqq-\sum M\left(\gamma_{\alpha}\right)=-M(\gamma) \text {. }
$$

We next prove the relations (4.45-4.47):

$(\alpha)$ Relation (4.45) follows since all lines of $\bar{\gamma}(U)$ are constant relative to $\gamma$ (Lemma 4.7). 
( $\beta$ ) Proof of relation (4.46). According to the hypothesis of induction we have

This implies

$$
\operatorname{degr}_{t} Y_{\gamma_{\alpha}}\left(K^{\gamma_{\alpha}}(K), q^{\gamma_{\alpha}}\right)<-M\left(\gamma_{\alpha}\right)
$$

For it is

$$
\operatorname{degr}_{t} t_{\gamma_{\alpha}} Y_{\gamma_{\alpha}}\left(K^{\gamma_{\alpha}}(K), q^{\gamma_{\alpha}}\right)<-M\left(\gamma_{\alpha}\right) \text {. }
$$

$$
\begin{gathered}
t_{\gamma_{\alpha}} Y_{\gamma_{\alpha}}=\left.t_{\varrho}^{d(\gamma)} Y_{\gamma_{\alpha}}\left(K^{\gamma_{\alpha}}, \varrho q^{\gamma_{\alpha}}\right)\right|_{\varrho=1}, \\
\operatorname{degr}_{t} t_{\varrho}^{d\left(\gamma_{\alpha}\right)} Y_{\gamma_{\alpha}}\left(K^{\gamma_{\alpha}}(K), \varrho q^{\gamma_{\alpha}}\right) \leqq \operatorname{degr}_{t} Y_{\gamma_{\alpha}}\left(K^{\gamma_{\alpha}}(K), \varrho q^{\gamma_{\alpha}}\right) .
\end{gathered}
$$

By definition of the substitution operator $\mathrm{S}_{\gamma}$

$$
\left(S_{\gamma} t^{\gamma_{\alpha}} Y_{\gamma_{\alpha}}\right)\left(K^{\gamma} q^{\gamma}\right)=\left(t^{\gamma_{\alpha}} Y_{\gamma_{\alpha}}\right)\left(K^{\gamma_{\alpha}}\left(K^{\gamma}\right), q^{\gamma_{\alpha}}\left(K^{\gamma}, q^{\gamma}\right)\right) .
$$

In $q^{\gamma \alpha}\left(K^{\gamma}, q^{\gamma}\right)$ only those $k_{a b \sigma}^{\gamma}$ occur with $L_{a b \sigma} \in \mathscr{L}(\bar{\gamma})$ which are constant on $H$ relative to $\gamma$. Hence

$$
\operatorname{degr}_{t}\left(S_{\gamma} t^{\gamma_{\alpha}} Y_{\gamma_{\alpha}}\right)\left(K^{\gamma}(K) q^{\gamma}\right)=\operatorname{degr}_{t}\left(t^{\gamma_{\alpha}} Y_{\gamma_{\alpha}}\right)\left(K^{\gamma_{\alpha}}(K), q^{\gamma_{\alpha}}\right) .
$$

$(\gamma)$ Proof of (4.47). Let $\gamma_{\alpha} \notin W$. According to the hypothesis of induction.

$$
\operatorname{degr}_{t, q^{\gamma_{\alpha}}} Y_{\gamma_{\alpha}}\left(K^{\gamma_{\alpha}}(K(T)), q^{\gamma_{\alpha}}\right) \leqq d\left(\gamma_{\alpha}\right)-M\left(\gamma_{\alpha}\right) .
$$

Applying Lemma 4.12

$$
\operatorname{degr}_{t}\left(1-t_{\gamma_{\alpha}}\right) Y_{\gamma_{\alpha}}\left(K^{\gamma_{\alpha}}(K), q^{\gamma_{\alpha}}\right)<-M\left(\gamma_{\alpha}\right) .
$$

Applying the substitution operator we obtain

$$
\left(S_{\gamma}\left(1-t_{\gamma_{\alpha}}\right) Y_{\gamma_{\alpha}}\right)\left(K^{\gamma}(K), q^{\gamma}\right)=\left(\left(1-t_{\gamma_{\alpha}} Y_{\gamma_{\alpha}}\right)\left(K^{\gamma_{\alpha}}(K), q^{\gamma_{\alpha}}\right)\left(K^{\gamma}(K), q^{\gamma}\right) .\right.
$$

Again $q^{\gamma \alpha}$ depends only on components $k_{a b \sigma}^{\gamma}$ of $K^{\gamma}$ which are constant on $H$.

Hence

$$
\left.\operatorname{degr}_{t}\left(S_{\gamma}\left(1-t_{\gamma_{\alpha}}\right) Y_{\gamma_{\alpha}}\right)\left(K^{\gamma}(K), q^{\gamma}\right)=\operatorname{degr}_{t}\left(1-t^{\gamma_{\alpha}}\right) Y_{\gamma_{\alpha}}\right)\left(K^{\gamma_{\alpha}}(K), q^{\gamma_{\alpha}}\right)
$$

and

$$
\operatorname{degr}_{t}\left(S_{\gamma}\left(1-t_{\gamma_{\alpha}}\right) Y_{\gamma_{\alpha}}\right)\left(K^{\gamma}(K), q^{\gamma}\right)<-M\left(\gamma_{\alpha}\right) .
$$

(ii) Case $\gamma \notin W$.

In that case the recursion formula reads

$$
Y_{\gamma}=I_{\gamma / \gamma_{1} \ldots \gamma_{c}} S_{\gamma}\left(-t_{\gamma_{1}}\right) Y_{\gamma_{1}} \ldots\left(-t_{\gamma_{c}}\right) Y_{\gamma_{c}} .
$$

The relations

$$
\begin{aligned}
& \operatorname{degr}_{t q^{\gamma}} I_{\gamma / \gamma_{1} \ldots \gamma_{\alpha}}=d(\bar{\gamma})-4 m(\bar{\gamma}), \\
& \operatorname{degr}_{t q^{\gamma}} S_{\gamma} t_{\gamma_{\alpha}} Y_{\gamma_{\alpha}} \leqq d\left(\gamma_{\alpha}\right)-M\left(\gamma_{\alpha}\right) \quad \text { if } \quad \gamma_{\alpha} \notin W, \\
& \operatorname{degr}_{t q^{\gamma}} S_{\gamma} t_{\gamma^{\alpha}} Y_{\gamma^{\alpha}}<d\left(\gamma_{\alpha}\right)-M\left(\gamma_{\alpha}\right) \text { if } \quad \gamma_{\alpha} \in W
\end{aligned}
$$


imply

$$
\begin{aligned}
\operatorname{degr}_{t q^{\gamma}} Y_{\gamma} & \leqq d(\bar{\gamma})-4 m(\bar{\gamma})+\sum_{\alpha}\left(d\left(\gamma^{\alpha}\right)-M\left(\gamma^{\alpha}\right)\right) \\
& \leqq d(\gamma)-M(\gamma)
\end{aligned}
$$

since $d(\bar{\gamma})+\sum d\left(\gamma_{\alpha}\right) \leqq d(\gamma)$.

(4.51) is the inequality (4.42) stated in the lemma.

We now prove the relations $(4.48-4.50)$.

$(\alpha)$ The relation (4.48) follows from the definitions of $I$.

$(\beta)$ The hypothesis of induction

implies

$$
\operatorname{degr}_{t q^{\gamma_{\alpha}}} Y_{\gamma^{\alpha}}\left(K^{\gamma_{\alpha}}(K), q^{\gamma_{\alpha}}\right) \leqq d\left(\gamma_{\alpha}\right)<M\left(\gamma_{\alpha}\right)
$$

$$
\operatorname{degr}_{t q^{\gamma_{\alpha}}} t^{\gamma_{\alpha}} Y_{\gamma_{\alpha}}\left(K^{\gamma_{\alpha}}(K), q^{\gamma_{\alpha}}\right) \leqq d\left(\gamma_{\alpha}\right)-M\left(\gamma_{\alpha}\right) \text {. }
$$

Application of the substitution operator $S_{\gamma}$ to $t^{\gamma_{\alpha}} Y_{\gamma_{\alpha}}$ yields

$$
\left(S_{\gamma} t^{\gamma_{\alpha}} Y_{\gamma_{\alpha}}\right)\left(K^{\gamma}(K), q^{\gamma}\right)=\left(t^{\gamma_{\alpha}} Y_{\gamma_{\alpha}}\right)\left(K^{\gamma_{\alpha}}(K), q^{\gamma_{\alpha}}\right)\left(K^{\gamma}(K), q^{\gamma}\right) .
$$

Since $t^{\gamma_{\alpha}} Y_{\gamma_{\alpha}}$ is a polynominal in $q^{\gamma_{\alpha}}$ the substitution

$$
q^{\gamma_{\alpha}} \rightarrow q^{\gamma_{\alpha}}\left(K^{\gamma}(K), q^{\gamma}\right)
$$

can only decrease the degree with respect to $T, q^{\gamma}$ $\operatorname{degr}_{t q^{\gamma}}\left(S_{\gamma} t^{\gamma_{\alpha}} Y_{\gamma_{\alpha}}\right)\left(K^{\gamma}(K), q^{\gamma}\right)$

$$
\leqq \operatorname{degr}_{t q^{\gamma_{\alpha}}}\left(t^{\gamma_{\alpha}} Y_{\gamma_{\alpha}}\right)\left(K^{\gamma_{\alpha}}(K), q^{\gamma_{\alpha}}\right) \leqq d\left(\gamma_{\alpha}\right)-M\left(\gamma_{\alpha}\right)
$$

$(\gamma)$ Proof of relation (4.50). We assume $\gamma_{\alpha} \in W$. According to the hypothesis of induction

$$
\operatorname{degr}_{t} Y_{\gamma_{\alpha}}\left(K^{\gamma_{\alpha}}(K), q^{\gamma_{\alpha}}\right)<-M\left(\gamma^{\alpha}\right) .
$$

This implies

$$
\begin{aligned}
\operatorname{degr}_{t q^{\gamma_{\alpha}}} t^{\gamma_{\alpha}} & Y_{\gamma_{\alpha}}\left(K^{\gamma_{\alpha}}(K), q^{\gamma_{\alpha}}\right)<d\left(\gamma_{\alpha}\right)-M\left(\gamma_{\alpha}\right), \\
\operatorname{degr}_{t q^{\gamma}}\left(S_{\gamma}\right. & \left.t_{\gamma_{\alpha}} Y_{\gamma_{\alpha}}\right)\left(K^{\gamma}(K), q^{\gamma}\right) \\
& =\operatorname{degr}_{t q^{\gamma}}\left(t_{\gamma_{\alpha}} Y_{\gamma_{\alpha}}\right)\left(K^{\gamma_{\alpha}}(K), q^{\gamma_{\alpha}}\right)\left(K^{\gamma}(K), q^{\gamma}\right) \\
& \leqq \operatorname{degr}_{t q^{\gamma} \alpha}\left(t_{\gamma_{\alpha}} Y_{\gamma_{\alpha}}\right)\left(K^{\gamma_{\alpha}}(K), q^{\gamma_{\alpha}}\right)<d\left(\gamma_{\alpha}\right)-M\left(\gamma_{\alpha}\right) .
\end{aligned}
$$

This completes the proof of the lemma.

The results obtained in Lemma 4.13 will now be used in order to show that the dimension of the integral (4.1) is negative. We have

$$
X=\left(1-t_{\Gamma}\right) Y_{\Gamma} .
$$

First let $\Gamma \in W$. Then

$$
\operatorname{degr}_{t} Y_{\Gamma}(K q)<-M(\Gamma)
$$


implies

$$
\operatorname{degr}_{t}\left(1-t_{\Gamma}\right) Y_{\Gamma}(K q)<-M(\Gamma) .
$$

Let $\Gamma \notin W$. Then

implies

$$
\operatorname{degr}_{t, q} Y_{\Gamma}(K q) \leqq d(\Gamma)-M(\Gamma)
$$

$$
\operatorname{degr}_{t}\left(1-t_{\Gamma}\right) Y_{\Gamma}(K q)<-M(\Gamma) .
$$

In any case

$$
\operatorname{degr}_{t} X<-M(\Gamma)
$$

and therefore

$$
\operatorname{degr}_{t} R_{\Gamma}(K q)<-M(\Gamma) .
$$

Since $M_{\Gamma}$ is the number of independent parameters of the hyperplane $H$ it follows

$$
\operatorname{dim} \int_{H} d k R_{\Gamma}<0 .
$$

This completes the proof of the theorem.

\section{Appendix}

We shortly indicate the proof of the following generalized form of Eq. (4.54)

$$
\operatorname{degr}_{t, M} R_{\Gamma}(K q)<-M(\Gamma)
$$

which is useful for checking the equivalence of Bogoliubov's original definition of the renormalized Feynman integral to the one used in this paper [17] $M$ denotes a subset

$$
M \leqq\left\{\mu_{a b \sigma}\right\}
$$

of the mass parameters.

First we note that under the hypothesis of Lemma 4.12

$$
\operatorname{degr}_{t M}\left(1-t_{q}^{d}\right) F \leqq \operatorname{degr}_{t M q} F-d-1
$$

can be derived which is a generalization of (4.33-4.34). The Eq. (4.43-4.44) can be generalized to

$$
\begin{aligned}
& \operatorname{degr}_{t M q^{\gamma}} Y_{\gamma}\left(K^{\gamma}(K), q^{\gamma}\right) \leqq d(\gamma)-M(\gamma) \text { for } \gamma \in U, \gamma \notin W, \\
& \operatorname{degr}_{t M} Y_{\gamma}\left(K^{\gamma}(K), q^{\gamma}\right)<-M(\gamma) \text { for } \gamma \in W .
\end{aligned}
$$

These relations are derived from

$$
\begin{array}{ll}
\operatorname{degr}_{t M} I_{\gamma / \gamma_{1} \ldots \gamma_{c}} \leqq 0, & \text { if } \quad \gamma_{\alpha} \notin W, \\
\operatorname{degr}_{t M} S_{\gamma} t^{\gamma_{\alpha}} Y_{\gamma_{\alpha}}<-M\left(\gamma_{\alpha}\right) & \text { if } \\
\operatorname{degr}_{t M} S_{\gamma}\left(1-t^{\gamma_{\alpha}}\right) Y_{\gamma_{\alpha}}<-M\left(\gamma_{\alpha}\right) & \gamma_{\alpha} \notin W
\end{array}
$$


if $\gamma \in W$ and from

$$
\begin{array}{lll}
\operatorname{degr}{ }_{t M q^{\gamma}} I_{\gamma / \gamma_{1} \ldots \gamma_{c}} \leqq d(\bar{\gamma})-4 m(\bar{\gamma}), & \\
\operatorname{degr}_{t M q^{\gamma}} S_{\gamma} t^{\gamma_{\alpha}} Y_{\gamma_{\alpha}} \leqq d\left(\gamma_{\alpha}\right)-M\left(\gamma_{\alpha}\right) & \text { if } \quad \gamma_{\alpha} \notin W, \\
\operatorname{degr}_{t M q^{\gamma}} S_{\gamma} t^{\gamma_{\alpha}} Y_{\gamma_{\alpha}}<d\left(\gamma_{\alpha}\right)-M\left(\gamma_{\alpha}\right) \text { if } \quad \gamma_{\alpha} \in W
\end{array}
$$

if $\gamma \notin W$. The result (A.1) follows if the Eqs. (A.3-A.4) are applied to (4.52).

I am grateful to Dr. K. Hepp for many useful discussions and I wish to thank Dr. G. Dell'Antonio and Dr. L. Motchane for the kind hospitality extended to me at the I.H.E.S. and the Istituto di Fisica Teorica.

\title{
Bibliography
}

1. Bogoliubov, N. N., and D. V. Shirkov: Introduction to the theory of quantized fields. New York: Interscience 1959.

2. -, and O. S. Parasiuk: Acta Math. 97, 227 (1957).

3. Hepp, K.: Commun. Math. Phys. 6, 161 (1967).

4. Speer, E.: J. Math. Phys. 9, 1404 (1968).

5. Zimmermann, W.: Commun. Math. Phys. 6, 161 (1967).

6. Dyson, F. J.: Phys. Rev. 75, 1736 (1949)

7. Salam, A.: Phys. Rev. 82, 217 (1951).

8. - Phys. Rev. 84, 426 (1951).

9. Wu, T. T.: Phys. Rev. 125, 1436 (1962).

10. Steinmann, O.: Ann. Phys. 36, 267 (1966).

11. Yennie, D. R.: Cargese Lectures (1967).

12. Caianiello, L. R., F. Guerra et M. Marinaro: Nuovo Cimento 60 A, 713 (1969).

13. Weinberg, S.: Phys. Rev. 118, 838 (1960).

14. Hahn, Y., and W. Zimmermann: Commun. Math. Phys. 10, 330 (1968).

15. Zimmermann, W.: Commun. Math. Phys. 11, 1 (1969).

16. See for instance Bjorken, J., and S. Drell: Relativistic quantum fields. New York: McGraw-Hill 1962.

17. Hepp, K., and W. Zimmermann: Unpublished.

\author{
W. Zimmermann \\ Courant Institute \\ New York University \\ 251, Mercer St. \\ New York, N. Y. 10012, USA
}

